McGill Law Journal

Revue de droit de McGill

\title{
Antoine Leduc, Mondialisation et harmonisation du droit des sûretés, Montréal, Thémis, 2012, 670 pages
}

\section{Laurence Bich-Carrière}

Volume 59, numéro 2, december 2013

URI : https://id.erudit.org/iderudit/1022314ar

DOI : https://doi.org/10.7202/1022314ar

Aller au sommaire du numéro

Éditeur(s)

McGill Law Journal / Revue de droit de McGill

ISSN

0024-9041 (imprimé)

1920-6356 (numérique)

Découvrir la revue

Citer ce compte rendu

Bich-Carrière, L. (2013). Compte rendu de [Antoine Leduc, Mondialisation et harmonisation du droit des sûretés, Montréal, Thémis, 2012, 670 pages]. McGill Law Journal / Revue de droit de McGill, 59(2), 477-480.

https://doi.org/10.7202/1022314ar d'utilisation que vous pouvez consulter en ligne.

https://apropos.erudit.org/fr/usagers/politique-dutilisation/ 
McGill Law Journal — Revue de droit de McGill

RECENSION-

Antoine Leduc, Mondialisation et harmonisation du droit des sûretés, Montréal, Thémis, 2012, 670 pages.

Version remaniée de la thèse de doctorat de l'auteur, réalisée sous la direction du professeur Pierre Ciotola ${ }^{1}$, l'ouvrage Mondialisation et harmonisation du droit des surretés ${ }^{2}$ propose une réflexion critique, empirique et comparative sur les conditions d'implantation d'un régime de sûretés moderne dans un contexte transnational.

Le point d'ancrage de l'étude est le constat d'une tendance à l'uniformisation des mécanismes de crédit garanti, compris comme nécessaires à la stabilité du commerce et propices à la coopération économique, et ce, tant entre les démocraties libérales que dans les pays en voie de développement, où elle est introduite par les organisations internationales. Cette tendance est dictée par des impératifs pratiques, mais comment se réalise-t-elle concrètement?

L'auteur aborde la question en deux temps. Au terme d'un examen des divers systèmes de la tradition juridique occidentale, il dégage d'abord une structure archétypale, la mécanique d'un modèle efficient (c'est l'étude systémique de la partie I de l'ouvrage). Ce modèle, autour duquel s'articulent les efforts de réforme des institutions de développement international, est ensuite confronté à la réalité des traditions juridiques, en l'occurrence non occidentales, de pays visés par ces efforts de réforme (c'est l'étude appliquée de la partie II). L'analyse se trouve ici nourrie des conclusions que l'auteur a pu former par sa participation à deux projets entrepris sous l'égide de l'Agence canadienne de développement international, l'un portant sur le crédit foncier en Égypte (une initiative commune avec la Banque mondiale) ${ }^{3}$, l'autre sur l'urbanisme, l'habitat et le crédit foncier en République démocratique du Congo4.

1 Antoine Leduc, De la réforme et de l'harmonisation du droit des sûretés dans un contexte de mondialisation de l'économie : vers un retour au paradigme de l'uniformisation du droit?, thèse de doctorat en droit, Université de Montréal, 2011 [non publiée; Leduc, Réforme].

2 Antoine Leduc, Mondialisation et harmonisation du droit des sûretés, Montréal, Thémis, 2010 [Leduc, Mondialisation et harmonisation].

$3 \quad$ Ibid à la $\mathrm{p} 443$.

$4 \quad$ Ibid à la $\mathrm{p} 432$. 
D'entrée de jeu, l'auteur postule qu'il existe en Occident une conception commune de l'ordre juridique, fondée sur une communauté de valeurs qu'il s'attelle à définir et à répertorier tout en identifiant les intérêts qu'on cherche à promouvoir et à protéger ${ }^{5}$. Il entreprend pour ce faire l'étude comparée du droit des sûretés dans plusieurs systèmes juridiques occidentaux, au premier plan desquels, ceux du Québec et des États-Unis et, dans une moindre mesure, du Canada anglais. Des références ponctuelles sont également faites aux droits français et anglais, à la fois en leur qualité de référents historiques du droit nord-américain et pour leur rapport à ce droit européen vers lequel ils évoluent. La comparaison est poussée à travers d'autres instruments transnationaux ou internationaux ${ }^{6}$, ainsi que par des références au droit de la faillite (domaine privilégié des priorités et sûretés) ${ }^{7}$.

Diverses techniques d'harmonisation sont en outre présentées et évaluées. Aux techniques dominantes, l'auteur semble préférer une approche plus souple ou moins monomodale: le modèle américain, note-t-il par exemple, a lancé la réflexion sur le droit des sûretés moderne et, s'il s'agit d'une codification efficace et sophistiquée, il pourrait encore s'inspirer des innovations de systèmes voisins, particulièrement du système québécois ${ }^{8}$.

L'analyse est ici d'une facture classique. Théorie du droit du crédit garanti (chapitre 1), évolution des mécanismes (chapitre 2), modalités (chapitre 3), techniques d'harmonisation et limites (chapitre 4), les thèmes abordés et leur ordonnancement sont ceux des ouvrages de droit des sûre-

$5 \quad$ Ibid à la p 13.

6 Voir par ex CNUDCI, Guide législatif de la CNUDCI sur le droit de l'insolvabilité, New York, NU, 2005; Loi type de la CNUDCI sur l'insolvabilité internationale, Rés AG 52/158, Doc off AG NU, 52 e sess, Doc NU A/RES/52/158 (1997); CNUDCI, Insolvabilité internationale: Guide pour l' incorporation de la Loi type de la CNUDCI sur l'insolvabilité internationale, 30 e sess, Doc NU A/CN.9/442 (1997); CNUDCI, Guide législatif de la CNUDCI sur les opérations garanties, New York, NU, 2011; FMI, Département juridique, Pour des procédures d'insolvabilité ordonnées et efficaces : Principales questions, Washington, FMI, 2000, en-ligne: <www.imf.org/external/pubs/ft/orderly/fre>; Banque mondiale, Principes et directives régissant le traitement de l'insolvabilité et la protection des droits des créanciers, Washington, Banque mondiale, 2001, en-ligne: $<$ www.worldbank.org/ifa/ipg_fre.pdf>; Banque mondiale, Principes régissant le traitement de l'insolvabilité et la protection des droits des créanciers, Washington, Banque mondiale, 2005, en-ligne : <www.worldbank.org/ifa/IPG_Revised_Principles_French.pdf>; Banque européenne pour la reconstruction et le développement, Model Law on Secured Transactions, Londres, BERD, 2004; OÉA, CIDIP-VII, Model Registry Regulations under the Model Inter-American law on Secured Transactions, Doc off OEA/Ser.K/XXI.7/ CIDIP-VII/doc.3/09 rev.3.

7 Leduc, Mondialisation et harmonisation, supra note 2 aux pp 76-121.

$8 \quad$ Ibid aux pp 292-300. 
tés. L'exposé est clair, technique, complet, parfois didactique, conférant à l'ouvrage le caractère d'un texte de référence.

Le mérite de cette première partie repose surtout sur son caractère méthodique. La seconde se distingue de son côté par son originalité et présente une analyse empirique par le biais de deux études de $\operatorname{cas}^{9}$. Une telle approche demeure somme toute assez rare dans les ouvrages juridiques, même en droit comparé, et elle est, à notre connaissance, inédite en ce qui concerne le droit des sûretés au Québec.

Se fondant sur des considérations socioculturelles et anthropologiques et revenant sur l'une des valeurs-clés du modèle occidental, l'auteur amorce alors une étude de la notion d' "État de droit " (chapitre 1), dans ses fondements historiques et théoriques, et telle qu'elle est comprise et pratiquée par les principaux bailleurs de fonds de l'aide internationale ${ }^{10}$. En bref, un régime de sûretés ne peut être efficace s'il n'est soutenu par des institutions traduisant " les principes de la primauté de l'individu et la reconnaissance du droit de propriété de l'individu dans ses biens, mis en œuvre grâce à l'État de droit $»^{11}$. Dès lors, ce que doivent viser les réformes, c'est l'implantation de méthodes de gouvernance solides, qui favoriseront le développement "organique» de ces principes, plutôt que l'imposition superficielle d'un régime coupé de ses fondements conceptuels. Si la convergence semblait presque naturelle dans un hémisphère nord-ouest aux systèmes prisant les mêmes valeurs, une transposition est-elle réellement possible à travers les traditions juridiques? C'est ici qu'interviennent les deux études de cas annoncées, l'Égypte d'abord (chapitre 2), puis la République démocratique du Congo (chapitre 3).

De son expérience africaine, l'auteur reste sceptique. Au-delà du manque d'infrastructure ou de la gestion inefficace de ressources souvent maigres, au-delà de la fragmentation des sources ou de l'importance de la coutume et de l'oralité, au-delà encore des problèmes d'accessibilité ou de cohérence qu'elles entraînent, ce sont des divergences dans la conception même du droit — et des valeurs qui le sous-tendent — qui font obstacle à la mise en œuvre efficace d'un régime de sûretés moderne ${ }^{12}$. En Égypte par exemple, les efforts de réforme des instances démocratiques et populaires sont en butte à une tradition juridico-religieuse qui suppose, ex-

$9 \quad$ La première étude de cas porte sur l'Egypte (ibid aux pp 439-536) et la seconde porte sur la République démocratique du Congo (ibid aux pp 537-76).

$10 \quad$ Ibid aux pp 359-438.

11 Leduc, Réforme, supra note 1 à la $\mathrm{p}$ iii. Cette idée est reprise en substance dans l'ouvrage recensé (Leduc, Mondialisation et harmonisation, supra note 2 aux pp 4-5).

12 Ibid aux pp 534-36, 575-76. 
plique l'auteur, un droit "révélé", "immuable" car d'origine divine ${ }^{13}$. Quant à la tradition congolaise, elle est le reflet d'une société où les intérêts collectifs priment les intérêts individuels : comment alors espérer implanter de façon viable un système qui suppose la protection des droits individuels de manière uniforme ${ }^{14}$ ?

Peut-on taxer ici l'auteur de pessimisme? Selon le portrait qu'il en trace, les pays étudiés ne semblent pas près d'une révolution paradigmatique juridique. Pourtant, l'économie moderne a montré certains bouleversements conceptuels étonnants de rapidité (pensons à la Chine, pour ne donner qu'un exemple ${ }^{15}$, que l'auteur, il est vrai, n'ignore pas) ${ }^{16}$. Quoi qu'il en soit, retour sur le postulat: le crédit garanti ne saurait s'implanter de manière viable sans un État de droit garant à la fois d'une structure institutionnelle, de la liberté des sujets de droit et du respect de leurs intérêts, notamment en matière de propriété privée. Si une certaine diversité peut être fertile - l'auteur rappelle le cas du Québec dans l'univers nord-américain ${ }^{17}$ - il doit toutefois s'agir d'une diversité dans l'application et non dans les fondements. Les principes de gouvernance libérale ne sont pas négociables, n'en déplaise à ceux qui ont érigé le "phénomène de la "diversité culturelle" anthropologique [...] au rang de "patrimoine commun de l'humanité" "18. Si le lecteur avait pu en douter jusque-là, l'auteur s'inscrit résolument en faux d'une certaine mouvance en matière de pluralisme juridique et affirme, s'appuyant sur son sentiment d'échec sur le terrain, l'aboutissement des valeurs libérales.

Outre une étude systématique des régimes de sûretés modernes - et de la manière de les conceptualiser - , la contribution de l'ouvrage réside dans la présentation d'une démonstration en règle de ce que leur mise en place n'est, sans institutions solides et solidement établies, que "fantasme $»^{19}$.

Laurence Bich-Carrière

13 Ibid aux pp 466-81, 577-78.

14 Ibid aux pp 543-53.

15 Voir par ex You-tien Hsing, The Great Urban Transformation: Politics of Land and Property in China, New York, Oxford University Press, 2010.

16 Leduc, Mondialisation et harmonisation, supra note 2 aux pp 429-31.

17 Ibid aux pp 344-45.

18 Ibid à la $\mathrm{p} 576$.

19 Ibid à la $\mathrm{p} 579$. 